\title{
Case Report: Report of 2 Different Cases of Ovarian Teratoma Evaluated by Dynamic Contrast-Enhanced Ultrasound
}

\author{
Katja Glutig ${ }^{1 *}$, Ilmi Alhussami ${ }^{2}$, Paul-Christian Krüger ${ }^{1}$, Matthias Waginger ${ }^{1}$, \\ Felicitas Eckoldt ${ }^{2}$ and Hans-Joachim Mentzel ${ }^{1}$
}

' Section for Pediatric Radiology, Institute for Diagnostic and Interventional Radiology, University Hospital Jena, Jena, Germany, ${ }^{2}$ Pediatric Surgery Clinic, University Hospital Jena, Jena, Germany

Ovarian masses are not easily differentiated on transabdominal ultrasound in children. A useful supplement in various pediatric applications is dynamic contrast-enhanced ultrasound (dynCEUS). It can be performed quickly and easily. However, the literature for dynCEUS on pediatric ovarian masses is limited. We compared two cases with ovarian teratoma in which dynCEUS was a helpful additional tool.

\section{OPEN ACCESS}

Edited by: Alessandro Inserra Bambino Gesù Children Hospital (IRCCS), Italy

Reviewed by:

Sherif Mohamed Shehata,

Tanta University, Egypt

Erik David Skarsgard

British Columbia Children's

Hospital, Canada

*Correspondence:

Katja Glutig

katja.g/utig@med.uni-jena.de

Specialty section

This article was submitted to

Pediatric Surgery,

a section of the journal

Frontiers in Pediatrics

Received: 16 March 2021 Accepted: 19 May 2021

Published: 11 June 2021

Citation:

Glutig K, Alhussami I, Krüger P-C

Waginger $M$, Eckoldt $F$ and

Mentzel H-J (2021) Case Report:

Report of 2 Different Cases of Ovarian

Teratoma Evaluated by Dynamic Contrast-Enhanced Ultrasound.

Front. Pediatr. 9:681404.

doi: 10.3389/fped.2021.681404
Keywords: teratoma, ovary, ultrasound, dynamic CEUS, pediatric

\section{INTRODUCTION}

Non-specific chronic to acute abdominal pain is the primary clinical symptom of ovarian masses that often lead to a visit to the emergency department. Transabdominal B-mode ultrasound (US) is then the initial imaging modality. Neoplasms of the ovaries in girls are rare, with an incidence of only 3 cases per 100,000 per year (1), although true neoplasms are occasionally difficult to distinguish from other ovarian changes on B-mode ultrasound. This may be a hemorrhagic ovarian cyst, endometriosis, or torsion. Another differential diagnosis is a conglomerate tumor involving the ovary after a perforated appendicitis (2). In almost all cases, surgery is then the treatment of choice, although it should be performed sparing the ovary and preserving fertility (3). It is obligatory to assess the pathology of ovarian masses in advance with imaging and laboratory findings. Additional clinical clues include elevated tumor markers, virilization, and pre-mature puberty (4). Subsequently, the required surgical approach should be carefully planned accordingly (5).

Additional magnetic resonance imaging (MRI) is helpful in differentiation due to the advantageous high contrast resolution for soft tissue. Therefore, MRI is considered the gold standard of modern diagnostics without ionizing radiation in the evaluation of pelvic masses (6). Diffusion-weighted imaging (DWI) and the use of MRI contrast media in dynamic MRI also improve diagnostic accuracy. However, in younger children, sedation or anesthesia is often required for successful MRI examinations to achieve artifact-free imaging. However, MRI examinations under anesthesia or sedation are time-consuming, personnel-intensive, and costly.

Time-resolved dynamic contrast-enhanced ultrasound (dynCEUS) is another diagnostic modality. Lately, the use of dynCEUS has been increasing significantly in pediatric cases. Dynamic CEUS is quick and easy to perform in pediatric patients and is very well-tolerated (7). Visual analysis of microbubble contrast media (SonoVue ${ }^{\circledR}$ ) distribution is possible during the examination. The intravenously applied microbubbles remain strictly intravascular and the gating of reflex microbubbles represents the vascularization and perfusion of a mass (8). Therefore, CEUS is well-suited to differentiate tumors, such as those of the ovaries. Several studies in adults describe 


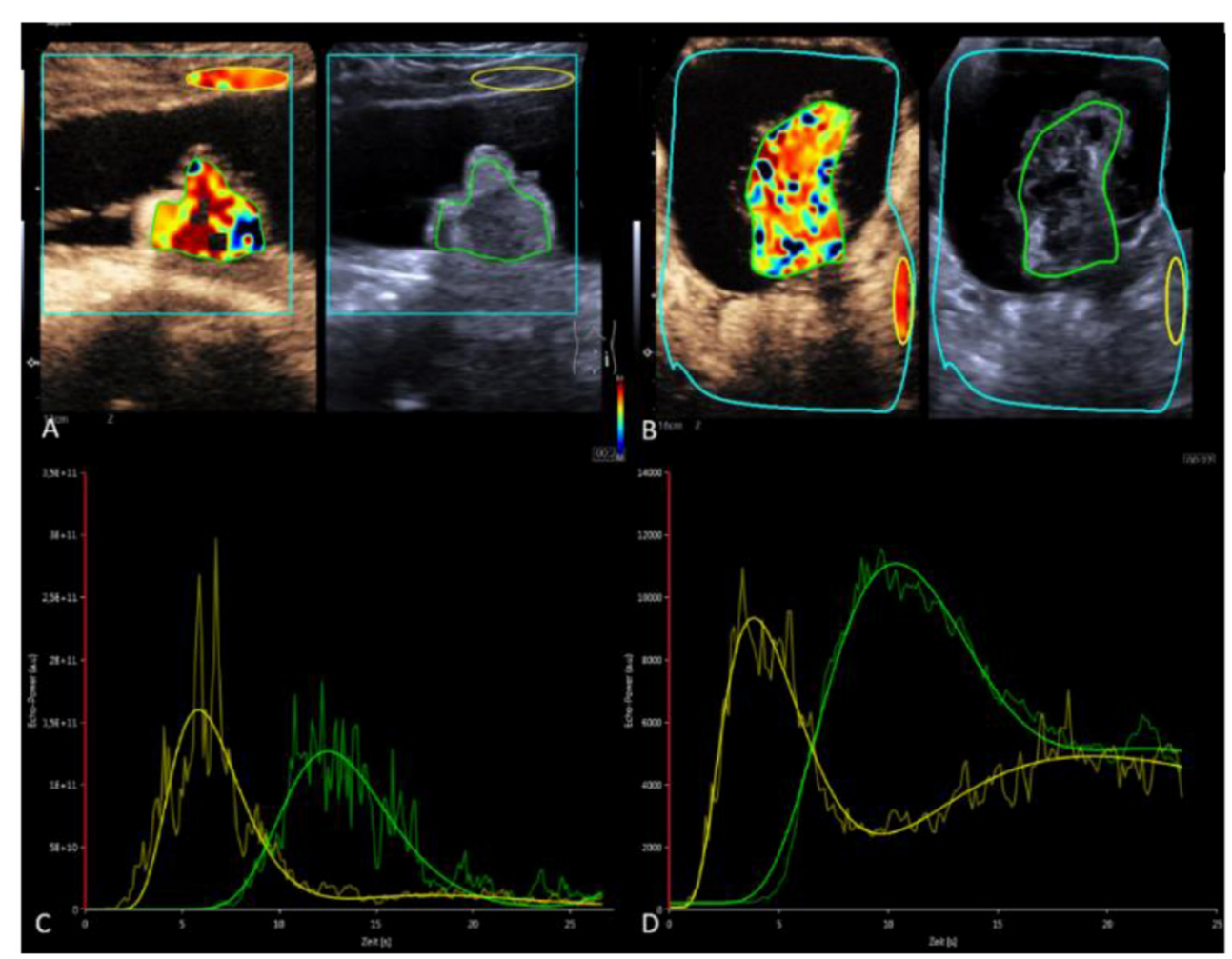

FIGURE 1 | Quantitative analysis using VueBoxTM software (Bracco Imaging, Germany). (A,B) Case 1: mature teratoma. (C,D) Case 2 Immature teratoma with potential malignant changes. (A,C) CEUS with contrast image (left) and B-mode (right) in parallel mode. Color coding of RT within the set ROI's. Green ROI in the solid portion of the teratomas. Yellow reference ROI in subcutaneous fat (A) or retroperitoneal (B). (C,D) Time-intensity curves-green curve: ROI measurement in the ovarian mass with visualization of PE, RT, TTP, AUC in case 1 and 2. Yellow curve: reference ROI.

the value of CEUS in differentiating benign from malignant ovarian tumors $(9,10)$. In contrast, the pediatric literature is limited (11). These two cases describe the findings on dynamic CEUS in pediatric patients and highlight the diagnostic differences in one case of a mature teratoma and another case of an immature teratoma with potential malignant changes. The aim of the work is to improve the understanding of dynamic CEUS in cases with of the ovary in children.

\section{MATERIALS AND METHODS Ultrasound Protocol}

Scans were performed with a Siemens (Sequoia, Siemens Healthineers, Erlangen, Germany) ultrasound machine by an experienced pediatric radiologist trained in CEUS with a 6$1 \mathrm{MHz}$ curvilinear transducer in both cases using the same abdominal preset. In childhood and adolescence, the ultrasound contrast media is applied depending on age $(0.1 \mathrm{ml} /$ year of life)

Abbreviations: AFP, Alpha-fetoprotein; CEUS, contrast-enhanced ultrasound; DWI, diffusion weighted imaging; dynCEUS, dynamic contrast-enhanced ultrasound; FDA, Food and Drug Administration; MRI, magnetic resonance imaging; PE, peak enhancement; RT, rise time; ROI, region of interest; TTP, time to peak; US, ultrasound; WiAUC, wash-in area under the curve; WiWoAUC, wash-in and wash-out area under the curve.
(7). Recently, the United States Food and Drug Administration (FDA) approved SonoVue ${ }^{\circledR}$ for intravenous application in pediatric liver imaging. The recommended dosage depends on the weight of the child. The dosage is $0.03 \mathrm{ml}$ per kg bodyweight with a maximum limit of $2.4 \mathrm{ml}$ per injection (12). For contrastenhanced ultrasound, our two cases received a standardized age-adapted amount of $0.1 \mathrm{ml}$ SonoVue ${ }^{\circledR}$ (Bracco Imaging, Germany) intravenously per year of life, followed by a $10 \mathrm{ml}$ bolus of $\mathrm{NaCl}$-solution.

One cycle of contrast was sufficient to characterize the lesion. For accurate quantitative evaluation of perfusion dynamics, a video clip of $\sim 120 \mathrm{~s}$ was recorded and stored. For software evaluation, the position of the transducer was not allowed to change with the onset of contrast media injection.

For quantitative perfusion analysis of dynamic CEUS, a dedicated software (VueBox ${ }^{\circledR}$, Bracco Imaging, Germany) was used (13). Time-intensity curves graphically represent the distribution of hyperreflective microbubbles in a lesion by placing a region of interest (ROI) in ellipsoidal or variable shape in the target lesion (Figure 1, green ROI) and another ROI in normal tissue (subcutaneous fat) as a reference (Figure 1, yellow ROI) (14). The evaluation software recorded parameters describing the enhancement, distribution, duration of enhancement, and washout of the bubbles. Peak enhancement (PE) as maximum 
TABLE 1 | Clinical characteristics, ultrasound data and dynamic CEUS parameters.

\begin{tabular}{lcc}
\hline & $\begin{array}{c}\text { Case 1 } \\
\text { Mature teratoma }\end{array}$ & $\begin{array}{c}\text { Case 2 } \\
\text { Immature teratoma }\end{array}$ \\
\hline Age, y & 16 & 10 \\
Weight, $\mathrm{kg}$ & 69 & 34 \\
Height, cm & 159 & 148 \\
Body mass index, $\mathrm{kg} / \mathrm{m}^{2}$ & 27.3 & 15.5 \\
AFP (serum), ng/nl & 2.1 & 15 \\
Beta-HCG (serum), mlU/ml & 1.2 & $<0.3$ \\
Amount SonoVue, $\mathrm{ml}$ & 1.6 & 1.0 \\
Ovary & 1 left & $1 \mathrm{eft}$ \\
Total, cm & $15.4 \times 5 \times 9.5 \times 12.3$ \\
Solid part, cm & $11.6 \times 5.3$ & $7.0 \times 4.2 \times 6.3$ \\
PE relative, \% & $3.0 \times 3.2$ & 117 \\
RT relative, \% & 79 & 223 \\
TTP relative, \% & 162 & 267 \\
WiAUC relative, \% & 124 & 259 \\
WiWoAUC relative, \% & 126 & 255 \\
\hline AFP Aloha-Fetoproten: & 119 & \\
\hline
\end{tabular}

AFP, Alpha-Fetoprotein; Beta-HCG, human chorionic gonadotropin; PE, peak enhancement; RT, rise time; TTP, time to peak; WiAUC, wash in Area under the curve; WiWoAUC, wash in und wash out Area under the curve; cm, centimeter; y, year; $\mathrm{kg}$, kilogram

signal intensity, time to peak (TTP) as the time to PE, rise time (RT), area under the curve at wash-in (WiAUC), and area under the curve at wash-in and wash-out (WiWoAUC) are the values with the strongest impact (15) (Table 1).

Following the German Drug Law, the case series was approved by the local ethics committee under registration number 20212102. Written informed consent was obtained from all patients and their custodial parents.

\section{CASES}

\section{Case 1: Mature Teratoma}

A 16-year-old girl was presented to the emergency department with severe abdominal pain that was crampy for several minutes. Anamnesis revealed weight loss of $4 \mathrm{~kg}$ in 2 weeks and recurrent nausea, hypertensive attacks, and occasional menstrual pain. All laboratory tests, including inflammatory and tumor markers, were normal. Menarche was at the age of 13 years and regular menstruation since then.

B-mode ultrasound and a multimodal MRI with DWI and contrast media showed a large cystic mass extending from the left ovary to $15.4 \mathrm{~cm}$. Within the thin-walled cyst were a hyperechoic solid structure of about $4.2 \mathrm{~cm}$ and a delicate septum (Figure 2). Doppler ultrasonography showed non-specific perfusion.

CEUS examination visually verified early arterial accumulation of vesicles in the solid structure. A small central portion of the solid structure remained free of microbubble contrast media throughout the examination. In the quantitative analysis TTP in the solid part was $12.5 \mathrm{~s}$, RT was $4.6 \mathrm{~s}$, and relative PE was 79\% (Figures $\mathbf{1 A}, \mathbf{C}$ ).
Based on the findings of multimodal MRI and dynamic CEUS, a diagnosis of mature teratoma was made, which was confirmed by laparoscopic surgery with unilateral tumor extirpation and histological workup. It was a mature teratoma without signs of malignancy.

\section{Case 2: Immature Teratoma With Potential Malignant Changes}

A 10-year-old girl with abdominal pain and a large abdominal mass palpable for a fortnight was presented. The tumor marker alpha-fetoprotein (AFP) in serum was slightly elevated at $15 \mathrm{ng} / \mathrm{ml}$. The other tumor markers and laboratory values were unremarkable. B-mode ultrasound and a multimodal MRI with DWI and contrast media visualized a cystic solid mass of a maximum of $15.5 \mathrm{~cm}$ depending on the left ovary. A contained irregularly configured solid portion without calcifications was $\sim 7.0 \mathrm{~cm}$ in size and with vigorous perfusion on Doppler ultrasonography (Figure 3). Analysis of the dynamic CEUS examination in the solid part showed a TTP of $10.4 \mathrm{~s}$ and an RT of 5.8 s, and relative PE was $117 \%$ (Figures 1B,D). Purely visual examination revealed very vigorous and irregular flooding of the bubble in the solid part (Figure 3C). The findings in multimodal MRI with contrast media suspected an immature teratoma with additional peritoneal tumor seeding. Therefore, after determination from the interdisciplinary tumor board, an abdominal laparotomy was performed. During surgery, no healthy ovarian tissue could be visually differentiated. Therefore, adnexal extirpation was performed. Intraoperatively, there was no peritoneal tumor seeding. Histologically, the findings of an ovarian teratoma were confirmed. In addition to mature cell differentiation, there were larger regions with immature cells with neuroectodermally differentiated parts in the sense of an immature teratoma in stage G1 according to Gonzalez-Crussi. There was no infiltration into the capsule. The removal was performed in healthy tissue (R0).

\section{DISCUSSION}

Based on the two different pediatric cases with an ovarian mass presented here, it can be shown that dynamic CEUS with subsequent quantitative analysis is a new diagnostic method that appears to be able to better differentiate lesions. An ovarian mass in girls rarely occurs and differentiation of this lesion is sometimes not straightforward (2). In this regard, a baseline transabdominal b-mode ultrasound and a complementary multimodal MRI with DWI and contrast media can well identify the characteristic features of ovarian masses (6). Both imaging methods can reliably determine tumor size, and also reliably distinguish cystic and solid parts contained therein (4).

Of particular importance in all diagnostic procedures is that vascularization is assessed, as it correlates with the invasive potential of a lesion (15). Thus, a high degree of vascularization and associated higher malignancy of a lesion is characterized by a dense network of small vessels. Also, perfusion is an important functional parameter and describes the vitality of a 

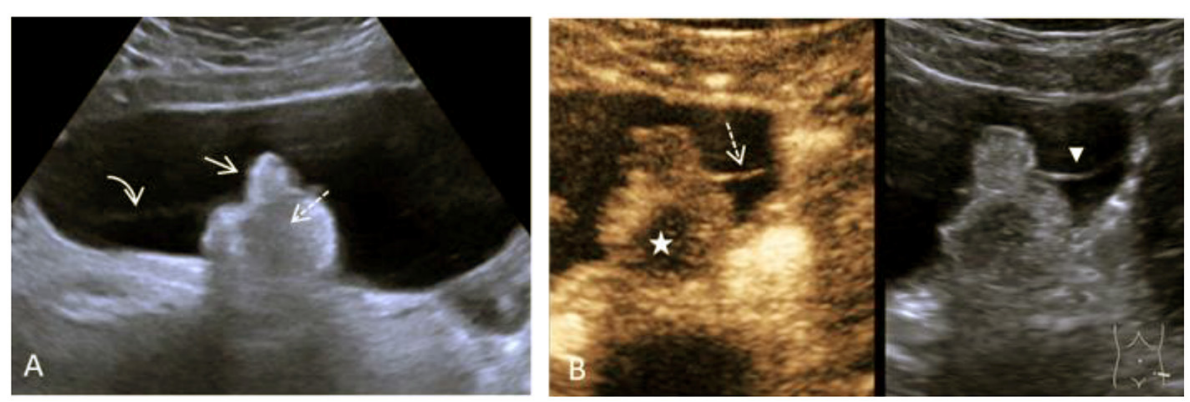

FIGURE 2 | Case 1, a 16-year-old girl with a mature teratoma of the right ovary. (A), B-mode Ultrasound showing a large cystic structure. In it, a solid lobulated portion, the so-called Rokitansky nodule, is adherent (arrow) to the dorsal wall. Within the solid echogenic mass, there are centrally echo-free areas with dorsal extinction (dashed arrow). There is very delicate septation between the solid part and the cyst wall (curved arrow). (B) CEUS of the solid part and the intracystic septation in the contrast image (left) and the native B-mode (right) in parallel mode. Image sequence $\sim 35 \mathrm{~s}$ after application of the contrast media i.v.: The intracystic membrane is very delicately detectable in the native B-mode (arrowhead) and accumulates contrast bubbles (dashed arrow). The contrast media shows strong perfusion with numerous microbubbles in the left part of the solid parts, the central part is excluded (star). In conjunction with the histological findings of the mature teratoma, this is a bony tooth.

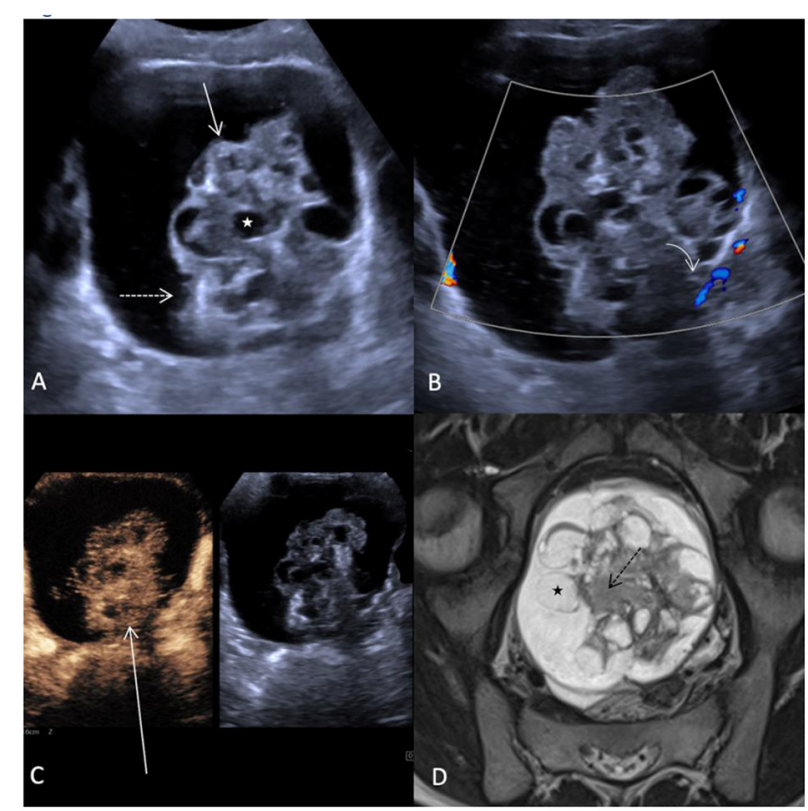

FIGURE 3 | Case 2, 10-year-old girl with an immature teratoma with potential malignant changes. (A) B-mode ultrasound showing a cystic structure extending from the left ovary up to $16 \mathrm{~cm}$ in diameter. Within the cyst there is a solid part (arrow) measuring up to $7.5 \mathrm{~cm}$. This is interspersed with an irregular surface (dashed arrow), inhomogeneous structure with multiple small cystoid lesions (white star). (B) In Doppler ultrasonography, no increased perfusion can be detected in the solid part (curved array). (C) Distribution pattern of contrast bubbles within the solid parts after 2 min. Strong enhancement with bizarre distribution within the irregularly configured solid mass (long arrow). (D) MRI of the pelvis shows the bizarre aspect of the solid mass with hyperintense cysts (black star) and irregularly configured hypointense stroma (black dashed arrow), (T2 TSE in paracoronal orientation).

tissue. Various physiological and pathological changes can alter the vascularization and perfusion of different tissues or tumors.
Doppler ultrasonography can visualize the vascularization of a lesion well, but the method is not sensitive enough to visualize perfusion even in very small vessels (16).

In contrast dynamic CEUS can visualize vascularization and perfusion in real-time using intravascular microbubbles. The echogenicity of the bubbles in a tissue corresponds to the volume of blood contained therein (17). According to Mehta et al., CEUS imaging can accurately assess neovascularization and better illuminate vascular abnormalities (8). Besides, special software can be used to objectively quantify various perfusion parameters. Thus, dynamic CEUS can help to differentiate between benign and malignant ovarian tumors in women by the purely visual, i.e., qualitative, assessment of a lesion as well as by the subsequent quantitative analysis (18).

Studies in adult women have already demonstrated the value of dynamic contrast-enhanced ultrasound in malignant ovarian tumors $(9,10)$. Malignant ovarian tumors showed higher peak enhancement (PE) intensity, delayed washout, and overall more enhancement with chaotic patterns than a benign lesion. Ultimately, CEUS was more specific than native B-scan or Doppler ultrasonography in differentiating ovarian carcinomas (19). Yang et.al also demonstrated in his study that ovarian carcinomas had increased perfusion values and area under the curve (AUC) was a significant value for diagnosis. AUC had the highest diagnostic confidence with a sensitivity of $87.3 \%$ and specificity of $80.8 \%$ (20).

To our knowledge, except from a single case reports (11), the diagnostic possibility of dynamic CEUS has not been currently reported in girls with an ovarian mass.

For example, Madenci et al. showed that girls who had in imaging a simple cyst in the ovary never associated it with malignancy (5). Predominantly solid ovarian tumors were malignant in $44 \%$ of cases and solid-cystic mixed tumors with elevated tumor markers were malignant in $40 \%$ of cases. Without elevated tumor markers, 5\% of cases were malignant if they were larger than $10 \mathrm{~cm}$ (5). Suspicious solid lesions in the ovary have a heterogeneous perfusion 
pattern on Doppler ultrasonography. In our experience and as well-demonstrated in the here described two cases, these heterogeneous patterns are much better visualized on CEUS than on Doppler ultrasonography.

Our case of an immature teratoma with potential malignant changes is in agreement with all these findings in adults and children. The tumor was very large, $16 \mathrm{~cm}$ in diameter. Dynamic CEUS and quantitative functional analysis showed significantly higher relative values for PE, RT, WiAUC, and WiWoAUC in the suspicious solid part compared to the first case of a mature teratoma. It must be remembered that the calculated absolute values of PE, WiAUC, and WiWaAUC are not well-comparable in children. Only TTP and RT can be evaluated as absolute values in seconds. We believe the reason for this is the dependence of the quantitative values on the amount of ultrasound contrast media applied (21). But the amount of contrast media varied sometimes significantly due to the different age and body weight of the examined girls. Yet the data of different ovarian masses is lacking in pediatric cases.

In our case, we assessed our decision to the operation management by the result of the MRI with contrast enhanced media and DWI. DynCEUS did not play a role in the treatment decision. The target for pediatric surgeons is always to perform an ovary sparing surgery (OSS) according to the modern surgery strategies (22). However, in the case presented here no healthy ovarian tissue could be visually differentiated during surgery. This was confirmed in the pathology, there was no normal healthy tissue to identify. Therefore, it was the only option to perform a unilateral tumor salpingo-oophorectomy.

We think that dynCEUS could be a helpful tool in differentiating unclear lesions on the ovary, including mature and immature teratomas. However, dynCEUS cannot be the only examination modality, it is always only one component in the overall imaging. In addition, a high level of experience in the use of contrast-enhanced ultrasound is required. Rather, dynamic CEUS could be quite helpful to increase the diagnostic confidence for the presence of immature teratoma. For this purpose, we assess the accumulation of the ultrasound contrast media in the ovary over time and the shape of the vascular architecture. Very bizarre vessels seem to be indicative of a more malignant lesion. Thus, in the second case of the girl with an immature teratoma showed a rather chaotic image during the contrast media inflow compared to the first case with a mature teratoma. This image corresponds to underlying vessels formed in malignant lesions by tumorangioneogenesis. Such tumor vessels, in contrast to nonpathological vessels, have different characteristics that have an impact on perfusion. Our data show that a longer absolute and relative RT exists in the solid part of the immature teratoma compared to the mature teratoma. The contrast

\section{REFERENCES}

1. Kaatsch P, Grabow D, Spix C. German Childhood Cancer Registry Annual Report 2018 (1980-2017). Mainz: Institute of Medical Biostatistics, Epidemiology and Informatics (IMBEI) at the University Medical Center of the Johannes Gutenberg University Mainz (2019). microbubbles in the immature teratoma take a longer time to reach maximal accumulation. The reduced flow velocity of contrast microbubbles in pathologic vessels may be due to the aging, corkscrew-like vessel shape, and branching of tumor endothelial cells across the vessel lumen (23). However, larger prospective studies are absolutely necessary to obtain more information and knowledge about this. Therefore, a prospective study with more patients would be helpful. This should be multicenter, as only a few comparable cases occur at one center within 1 year.

\section{SUMMARY-CONCLUSION}

These two cases teratoma demonstrate that dynamic contrastenhanced ultrasound with quantitative analysis can help differentiate ovarian masses in children and adolescents. Dynamic CEUS meets the requirements for imaging that is easy and quick to perform. It can be a helpful additional tool in the differentiation of tumors. Thus, it can have a complementary contribution in the planning of a ovarian sparing surgery. However, further pediatric studies of quantitative CEUS are needed to characterize typical patterns of findings, receive more data and to improve pre-operative predictive accuracy in the evaluation of ovarian lesions.

\section{DATA AVAILABILITY STATEMENT}

The original contributions presented in the study are included in the article/supplementary material, further inquiries can be directed to the corresponding author.

\section{ETHICS STATEMENT}

The studies involving human participants were reviewed and approved by Ethikkommission Universitätsklinikum Jena (2021-2102). Written informed consent to participate in this study was provided by the participants' legal guardian/next of kin.

\section{AUTHOR CONTRIBUTIONS}

All authors listed have made a substantial, direct and intellectual contribution to the work, and approved it for publication.

\section{FUNDING}

We acknowledge support by the German Research Foundation and the Open Access Publication Fund of the Thueringer Universitaets- und Landesbibliothek Jena Projekt-Nr. 433052568.

2. Ruttenstock EM, Saxena AK, Schwinger W, Sorantin E, Hoellwarth ME. Pediatric ovarian tumors-dilemmas in diagnosis and management. Eur J Pediatr Surg. (2010) 20:116-20. doi: 10.1055/s-0029-1246198

3. Luczak J, Baglaj M. Ovarian teratoma in children: a plea for collaborative clinical study. J Ovarian Res. (2018) 11:75. doi: 10.1186/s13048-0180448-2 
4. Lala SV, Strubel N. Ovarian neoplasms of childhood. Pediatr Radiol. (2019) 49:1463-75. doi: 10.1007/s00247-019-04456-8

5. Madenci AL, Vandewalle RJ, Dieffenbach BV, Laufer MR, Boyd TK, Voss SD, et al. Multicenter pre-operative assessment of pediatric ovarian malignancy. $J$ Pediatr Surg. (2019) 54:1921-5. doi: 10.1016/j.jpedsurg.2019.02.019

6. Darge K, Anupindi SA, Jaramillo D. MR imaging of the abdomen and pelvis in infants, children, and adolescents. Radiology. (2011) 261:1229. doi: 10.1148/radiol.11101922

7. Stenzel M, Mentzel HJ. Ultrasound elastography and contrast-enhanced ultrasound in infants, children and adolescents. Eur J Radiol. (2014) 83:15609. doi: 10.1016/j.ejrad.2014.06.007

8. Mehta KS, Lee JJ, Taha AG, Avgerinos E, Chaer RA. Vascular applications of contrast-enhanced ultrasound imaging. J Vasc Surg. (2017) 66:26674. doi: 10.1016/j.jvs.2016.12.133

9. Fleischer AC, Lyshchik A, Andreotti RF, Hwang M, Jones, HW, III, Fishman DA. Advances in sonographic detection of ovarian cancer: depiction of tumor neovascularity with microbubbles. AJR Am J Roentgenol. (2010) 194:3438. doi: 10.2214/AJR.09.3446

10. Ma X, Zhao Y, Zhang B, Ling W, Zhuo H, Jia H, et al. Contrast-enhanced ultrasound for differential diagnosis of malignant and benign ovarian tumors: systematic review and meta-analysis. Ultrasound Obstet Gynecol. (2015) 46:277-83. doi: 10.1002/uog.14800

11. Svensson JF, Larsson A, Uusijarvi J, Von Sivers K, Kaiser S. Oophoropexy, hyperbaric oxygen therapy, and contrast-enhanced ultrasound after asynchronous bilateral ovarian torsion. J Pediatr Surg. (2008) 43:1380-4. doi: 10.1016/j.jpedsurg.2008.02.024

12. Sidhu PS, Cantisani V, Dietrich CF, Gilja OH, Saftoiu A, Bartels E, et al. The EFSUMB guidelines and recommendations for the clinical practice of contrast-enhanced ultrasound (CEUS) in non-hepatic applications: update 2017 (Long Version). Ultraschall Med. (2018) 39:e2-e44. doi: 10.1055/a-0586-1107

13. Knieling F, Strobel D, Rompel O, Zapke M, Menendez-Castro C, Wolfel M, et al. Spectrum, applicability and diagnostic capacity of contrast-enhanced ultrasound in pediatric patients and young adults after intravenous application-a retrospective trial. Ultraschall Med. (2016) 37:61926. doi: 10.1055/s-0042-108429

14. Greis C. Quantitative evaluation of microvascular blood flow by contrast-enhanced ultrasound (CEUS). Clin Hemorheol Microcirc. (2011) 49:137-49. doi: 10.3233/CH-20111464
15. Wiesinger I, Kroiss E, Zausig N, Hornung M, Zeman F, Stroszczynski C, et al. Analysis of arterial dynamic micro-vascularization with contrast-enhanced ultrasound (CEUS) in thyroid lesions using external perfusion software: first results. Clin Hemorheol Microcirc. (2016) 64:747-55. doi: 10.3233/CH-168044

16. Jenderka KV, Delorme S. Principles of doppler sonography. Radiologe. (2015) 55:593-609; quiz 610. doi: 10.1007/s00117-015-2869-x

17. Rafailidis V, Deganello A, Watson T, Sidhu PS, Sellars ME. Enhancing the role of paediatric ultrasound with microbubbles: a review of intravenous applications. Br J Radiol. (2017) 90:20160556. doi: 10.1259/bjr. 20160556

18. Qiao JJ, Yu J, Yu Z, Li N, Song C, Li M. Contrast-enhanced ultrasonography in differential diagnosis of benign and malignant ovarian tumors. PLOS ONE. (2015) 10:e0118872. doi: 10.1371/journal.pone.0118872

19. Liu Z, Yang F, Zhang Y, Yu H, Zhu H, Yang R, et al. Conventional, doppler and contrast-enhanced ultrasonography in differential diagnosis of ovarian masses. Cell Physiol Biochem. (2016) 39:2398-408. doi: 10.1159/000 452508

20. Yang F, Yang TZ, Tian T, Wang JX, Luo H. Perfusion imaging of ovarian masses with contrast-enhanced ultrasonography (in Chinese). Sichuan Da Xue Xue Bao Yi Xue Ban. (2018) 49:587-93.

21. Emanuel AL, Meijer RI, Van Poelgeest E, Spoor P, Serne EH, Eringa EC. Contrast-enhanced ultrasound for quantification of tissue perfusion in humans. Microcirculation. (2020) 27:e12588. doi: 10.1111/micc.12588

22. Zhai A, Axt J, Hamilton EC, Koehler E, Lovvorn, HN, III. Assessing gonadal function after childhood ovarian surgery. J Pediatr Surg. (2012) 47:12729. doi: 10.1016/j.jpedsurg.2012.03.038

23. Dudley AC. Tumor endothelial cells. Cold Spring Harb Perspect Med. (2012) 2:a006536. doi: 10.1101/cshperspect.a006536

Conflict of Interest: The authors declare that the research was conducted in the absence of any commercial or financial relationships that could be construed as a potential conflict of interest.

Copyright (c) 2021 Glutig, Alhussami, Krüger, Waginger, Eckoldt and Mentzel. This is an open-access article distributed under the terms of the Creative Commons Attribution License (CC BY). The use, distribution or reproduction in other forums is permitted, provided the original author(s) and the copyright owner(s) are credited and that the original publication in this journal is cited, in accordance with accepted academic practice. No use, distribution or reproduction is permitted which does not comply with these terms. 